\title{
Gastrointestinal and Nongastrointestinal Complications of Esophagogastroduodenoscopy and Colonoscopy in the Real World: A Nationwide Standard Cohort Using the Common Data Model Database
}

\author{
Ha II Kim ${ }^{1}$, Jin Young Yoon ${ }^{1,2}$, Min Seob Kwak ${ }^{1,2}$, and Jae Myung Cha ${ }^{1,2}$ \\ ${ }^{1}$ Division of Gastroenterology, Department of Internal Medicine, Kyung Hee University Hospital at Gangdong, and 2Division of \\ Gastroenterology, Department of Internal Medicine, Kyung Hee University School of Medicine, Seoul, Korea
}

\section{Article Info}

Received July 19, 2020

Revised September 9, 2020

Accepted September 12, 2020

Published online January 8, 2021

\section{Corresponding Author}

Jae Myung Cha

ORCID https://orcid.org/0000-0001-9403-230X

E-mail drcha@khu.ac.kr

\begin{abstract}
Background/Aims: The global trend of an expanding aged population has increased concerns about complications correlated with gastrointestinal $(\mathrm{Gl})$ endoscopy in elderly patients; however there have been few reports published on this issue.

Methods: In this retrospective, observational cohort study performed between 2012 and 2017 , serious complications of esophagogastroduodenoscopy (EGD), colonoscopy, and colonoscopic polypectomy were compared between patients according to age ( $\geq 65$ years vs $18-64$ years). We used the Health Insurance Review and Assessment-National Patient Samples database, previously converted to the standardized Observational Medical Outcomes Partnership-Common Data Model. Serious complications within 30 days of the procedure included both $\mathrm{GI}$ complications (bleeding and perforation) and non-GI complications (cerebrovascular accident [CVA], acute myocardial infarction [AMI], congestive heart failure [CHF], and death).

Results: A total of 387,647 patients who underwent EGD, 241,094 who underwent colonoscopy, and 89,059 who underwent colonoscopic polypectomy were assessed as part of this investigation. During the study period, endoscopic procedures in the older group steadily increased in number in all endoscopy groups (all $p<0.001$ ). Further, pooled complication rates of bleeding, CVA, AMI, CHF, and death were approximately three times higher among older patients who underwent EGD or colonoscopy. Moreover, pooled complication rates of CVA, AMI, CHF, and death were approximately 2.2 to 5.0 times higher among older patients who underwent colonoscopic polypectomy.

Conclusions: Elderly patients experienced approximately three times more $\mathrm{Gl}$ and non-GI complications after EGD or colonoscopy than young patients. Physicians should pay attention to the potential risks of GI endoscopy in elderly patients. (Gut Liver 2021;15:569-578)
\end{abstract}

Key Words: Esophagogastroduodenoscopy; Colonoscopy; Complications; Population; Common Data Model

\section{INTRODUCTION}

Esophagogastroduodenoscopy (EGD) and colonoscopy are commonly performed gastrointestinal (GI) procedures that carry a low risk of complications. Most previous studies have focused on assessing GI complications of EGD and colonoscopy, ${ }^{1,2}$ while only a few have analyzed non-GI complications. ${ }^{3-6}$ As complications of EGD and colonosco- py are very rare, a large number of cases may be necessary to capture the exact rates of GI and non-GI complications associated with these procedures. Therefore, only population-based research may yield the exact magnitude of complications associated with EGD and colonoscopy. Yet, few population-based studies have reported on the complications of EGD and colonoscopy. ${ }^{4,7}$ In a prospective audit of upper GI endoscopy, the mortality and morbidity rates of 
diagnostic EGD were $1 / 2,000$ and $1 / 200$, respectively. ${ }^{8}$ In a single-center study, only two complications were reported from 12,841 diagnostic EGDs. ${ }^{9}$ After outpatient EGD, 2.5\% of patients required an emergency department or physician visit, and $1.1 \%$ required hospitalization within 30 days. ${ }^{10}$ The Clinical Outcomes Research Initiative database showed a cardiopulmonary event rate of $1 / 170$ and a mortality rate of $1 / 10,000$ after 140,000 upper GI procedures. ${ }^{3}$ In a systemic review of 57,742 screening colonoscopies, the pooled rate of overall complications was $2.8 / 1,000$ procedures. ${ }^{7}$ In the United States, the complication rate from mostly diagnostic colonoscopies was $5 / 1,000$ procedures. ${ }^{11}$ A recent systematic review reported a perforation rate of $4 / 10,000$ and major bleeding rate of $8 / 10,000$ associated with screening colonoscopy. ${ }^{1}$ The variable rates of complications associated with EGD and colonoscopy in previous studies $^{3-5}$ may be explained by differences in data source, patient population, healthcare resources, follow-up duration, and definitions of complications. Further, most previous studies were conducted in Western countries, ${ }^{1,5-7,12}$ and the complication rates of EGD and colonoscopy in Asian countries may be different from those of Western countries. Gathering exact knowledge on the expected complications of EGD and colonoscopy in the real world may help to minimize development of those complications.

Recently, the use of EGD and colonoscopy in elderly patients has been increasing as the diagnostic role of GI endoscopy is growing among this population. This trend likely increases the potential complications of GI endoscopy, but we currently have little information regarding their safety in elderly patients. In a recent population-based study, old age was an independent risk factor for cardiocerebrovascular complications after EGD and colonoscopy. ${ }^{4}$ Knowledge of potential complications of GI endoscopy in elderly patients may facilitate to careful selection of patients for appropriate indications and enable the operator to be familiar with the planned procedure and prepared to manage any complications that may arise in elderly patients. Therefore, a population-based study including elderly patients is necessary to assess the exact magnitudes of GI and non-GI complications associated with EGD and colonoscopy in the real world.

In this context, we sought to assess the GI and non-GI complications of EGD and colonoscopy in the real world using a nationwide standard cohort paired with the Common Data Model (CDM) database.

\section{MATERIALS AND METHODS}

\section{Data source}

We used the Health Insurance Review and AssessmentNational Patient Samples (HIRA-NPS) database from 2012 to 2017, previously converted to the standardized Observational Medical Outcomes Partnership-CDM. The HIRA is a repository of claims data collected during the process of reimbursing healthcare providers for services funded and covers all citizens in South Korea under the universal coverage system. ${ }^{13}$ The database contains comprehensive and rich information pertaining to healthcare services, such as treatments, pharmaceuticals, procedures, and diagnoses, for almost 50 million beneficiaries in South Korea. ${ }^{13}$ However, HIRA data have limitations in terms of accessibility and convenience to secure timely data, because the database's annual size is about one billion people or more. Therefore, sample data (i.e., HIRA-NPS) of HIRA were compiled to overcome these limitations. HIRA-NPS is a $3 \%$ representation of the national patient sample (encompassing about 1,000,000 people) per year extracted using a stratified randomized sampling method according to sex and age group at the patient level. It includes about 13\% (approximately 700,000) of inpatient and 1\% (approximately 400,000) of outpatient data, including all medical claims and prescriptions data for 1 year, which are calculated under the assumption of acceptable sampling error range and normal distribution for the optimal size of HIRA-NPS

However, it is difficult to characterize or analyze the data from HIRA-NPS and each hospital's data (especially international hospital data) in the same way or using the same tools because healthcare datasets are stored in databases that are built using a wide variety of data models and, often, local terminologies. ${ }^{14,15}$ An analysis across multiple disparate databases must either be tailored to accommodate each of the underlying data models and terminologies or convert the databases to a CDM. ${ }^{14}$ Recently, HIRA-NPS was converted to the Observational Medical Outcomes Partnership-CDM, in which disparate coding systems can be harmonized to a standardized vocabulary with minimal information loss. ${ }^{14,15}$ Variable terms for HIRA-NPS, which are typically expressed in nonstandard terms, were redeveloped into standard concepts using mapping. As HIRA data were initially recorded using the International Classification of Diseases, ninth revision codes, they were mapped to Systematized Nomenclature for Medicine-Clinical Terms codes in CDM data. ${ }^{14}$ As the Observational Medical Outcomes Partnership-CDM version of the HIRA-NPS database is provided as an open source in South Korea, it has advantages over the original HIRA-NPS database, which 
was limited by a preapproval for use, a usage fee, and limited use within a predetermined platform for online or offline use. This study was approved by the Institutional Review Board of Kyung Hee University (IRB number: 202004-043), and informed consent was waived.

\section{Study design}

We conducted a retrospective, observational cohort study to address 30-day GI and non-GI complications in patients 18 years or older undergoing outpatient EGD, colonoscopy, or colonoscopic polypectomy between January 1, 2012 and December 31, 2017. We described the demographic characteristics of the study population with each procedure by index year. The incidence rate of serious complications within 30 days of the endoscopy procedure was calculated as number of cases and proportion per 10,000 people by index year according to the type of endoscopy. Variables used in this study were age, age group
(18 to 64 years vs $\geq 65$ years), sex, and index year (2012 to 2017). Elderly patients were defined as those 65 years of age and older according to endoscopic practice guidelines for the elderly. ${ }^{16}$

\section{Definition of cohorts and complications}

The EGD cohort was defined as an outpatient population aged 18 years or older who underwent diagnostic EGD for the first person's history under the medical insurance program and no colonoscopy or colonoscopic polypectomy within \pm 30 days. For subjects who underwent multiple EGD procedures, the first was considered for analysis. To exclude other invasive endoscopy procedures such as enteroscopy or endoscopic retrograde cholangiopancreatography, only EGD procedures that were not on the same day or within \pm 30 days of these other procedures were included. EGD procedures with therapeutic interventions were also excluded from the EGD cohort. The colonoscopy

Table 1. Demographic Characteristics of the Study Population According to EGD, Colonoscopy, or Colonoscopic Polypectomy by Index Year

\begin{tabular}{|c|c|c|c|c|c|c|}
\hline Demographic characteristics & 2012 & 2013 & 2014 & 2015 & 2016 & 2017 \\
\hline \multicolumn{7}{|l|}{ Diagnostic EGD } \\
\hline No. of patients & 73,204 & 68,091 & 64,618 & 60,364 & 61,085 & 60,285 \\
\hline Age, yr & $49.9 \pm 15.6$ & $50.3 \pm 15.8$ & $48.6 \pm 15.7$ & $51.0 \pm 16.1$ & $51.0 \pm 16.3$ & $53.9 \pm 16.2$ \\
\hline Male sex & $34,027(46.5)$ & $32,265(47.4)$ & $30,106(46.6)$ & $28,692(47.5)$ & $28,990(47.5)$ & $28,761(47.7)$ \\
\hline \multicolumn{7}{|l|}{ Age group } \\
\hline Young (18-64 yr) & $58,534(80.0)$ & $53,651(78.8)$ & $53,079(82.1)$ & $46,523(77.1)$ & $46,856(76.7)$ & 39,607 (65.7) \\
\hline Age & $44.4 \pm 12.2$ & $44.5 \pm 12.3$ & $43.7 \pm 12.6$ & $44.6 \pm 12.5$ & $44.5 \pm 12.6$ & $45.0 \pm 11.5$ \\
\hline Male sex & $27,365(46.8)$ & $25,388(47.3)$ & $24,700(46.5)$ & $21,990(47.3)$ & $21,951(46.8)$ & 20,896 (52.8) \\
\hline Old ( $\geq 65 \mathrm{yr})$ & $14,670(20.0)$ & $14,440(21.2)$ & $11,539(17.9)$ & $13,841(22.9)$ & $14,229(23.3)$ & 20,678 (34.3) \\
\hline Age & $71.6 \pm 4.9$ & $71.8 \pm 5.1$ & $71.3 \pm 4.7$ & $72.2 \pm 5.3$ & $72.4 \pm 5.3$ & $72.1 \pm 5.8$ \\
\hline Male sex & $6,670(45.5)$ & $6,881(47.7)$ & $5,404(46.8)$ & $6,708(48.5)$ & $7,036(49.4)$ & $12,417(60.0)$ \\
\hline \multicolumn{7}{|l|}{ Diagnostic colonoscopy } \\
\hline No. of patients & 40,489 & 38,398 & 36,829 & 38,488 & 42,840 & 44,050 \\
\hline Age, yr & $52.0 \pm 12.2$ & $52.6 \pm 12.4$ & $50.9 \pm 12.3$ & $53.5 \pm 12.5$ & $54.1 \pm 12.6$ & $57.0 \pm 12.7$ \\
\hline Male sex & $21,225(52.4)$ & $19,754(51.4)$ & $19,074(51.8)$ & $19,942(51.8)$ & $22,145(51.7)$ & 22,870 (51.9) \\
\hline \multicolumn{7}{|l|}{ Age group } \\
\hline Young (18-64 yr) & 34,007 (84.0) & $31,546(82.2)$ & $31,676(86.0)$ & $30,798(80.0)$ & $33,598(78.4)$ & $28,862(65.5)$ \\
\hline Age & $48.6 \pm 10.0$ & $44.8 \pm 10.0$ & $47.8 \pm 10.3$ & $49.2 \pm 10.0$ & $49.6 \pm 10.0$ & $50.0 \pm 9.7$ \\
\hline Male sex & $17,730(52.1)$ & $16,105(51.1)$ & $16,406(51.8)$ & $15,816(51.4)$ & $17,253(51.4)$ & $16,374(56.7)$ \\
\hline Old ( $\geq 65 \mathrm{yr})$ & $6,482(16.0)$ & $6,852(17.8)$ & $5,153(14.0)$ & $7,690(20.0)$ & $9,242(21.6)$ & 15,188 (34.5) \\
\hline Age & $70.2 \pm 4.3$ & $70.3 \pm 4.5$ & $69.9 \pm 4.1$ & $70.4 \pm 4.6$ & $70.5 \pm 4.6$ & $70.5 \pm 5.3$ \\
\hline Male sex & $2,986(46.1)$ & 3,204 (46.8) & $2,486(48.2)$ & $3,567(46.4)$ & $4,353(47.1)$ & 8,251 (54.3) \\
\hline \multicolumn{7}{|l|}{ Colonoscopic polypectomy } \\
\hline No. of patients & 13,133 & 13,578 & 13,756 & 14,944 & 16,443 & 17,205 \\
\hline Age, yr & $55.7 \pm 10.9$ & $56.0 \pm 11.1$ & $54.2 \pm 11.1$ & $56.6 \pm 11.2$ & $57.1 \pm 11.2$ & $59.9 \pm 11.3$ \\
\hline Male sex & 8,689 (66.2) & 8,922 (65.7) & $8,807(64.0)$ & 9,514 (63.7) & 10,475 (63.7) & 10,887 (63.3) \\
\hline \multicolumn{7}{|l|}{ Age group } \\
\hline Young (18-64 yr) & $10,136(77.2)$ & $10,327(76.1)$ & 11,185 (81.3) & 11,101 (74.3) & $12,064(73.4)$ & 9,956 (57.9) \\
\hline Age & $51.5 \pm 8.4$ & $51.5 \pm 8.5$ & $50.6 \pm 8.8$ & $51.8 \pm 8.4$ & $52.2 \pm 8.4$ & $52.1 \pm 7.8$ \\
\hline Male sex & $6,831(67.4)$ & $6,864(66.5)$ & $7,240(64.7)$ & $7,226(65.1)$ & $7,791(64.6)$ & $7,326(73.6)$ \\
\hline Old ( $\geq 65 \mathrm{yr}$ ) & $2,997(22.8)$ & 3,251 (23.9) & $2,571(18.7)$ & $3,843(25.7)$ & $4,379(26.6)$ & $7,249(42.1)$ \\
\hline Age & $70.1 \pm 4.2$ & $70.2 \pm 4.4$ & $69.8 \pm 4.0$ & $70.4 \pm 4.5$ & $70.7 \pm 4.7$ & $70.6 \pm 5.3$ \\
\hline Male sex & 1,858 (62.0) & 2,058 (63.3) & $1,568(61.0)$ & $2,290(59.6)$ & 2,684 (61.3) & $5,139(70.9)$ \\
\hline
\end{tabular}

Data are presented as mean \pm SD or number $(\%)$.

EGD, esophagogastroduodenoscopy. 
cohort was defined in the same way as the diagnostic EGD cohort as patients who underwent an outpatient diagnostic colonoscopy for the first person's history under the medical insurance program and who underwent colonoscopy with or without biopsy. Finally, the colonoscopic polypectomy cohort was also defined in the same way and included patients who underwent single or multiple polypectomies and mucosal resections; however, it excluded cases of submucosal dissection or other therapeutic interventions. The strategies for treating colorectal polyp in Korea are similar to those in Western countries, ${ }^{17}$ that is, diminutive polyps $<5 \mathrm{~mm}$ are removed by forceps biopsy and polyps $\geq 5 \mathrm{~mm}$ are removed by snare polypectomy. Concept identification and concept codes of concept sets for endoscopy are described in Supplementary Table 1.

Serious 30-day complications were defined as complications occurring within 30 days of initial procedures and presenting to an emergency department or inpatient visit based on the standard 30-day period in the postsurgical literature and a previous population-based study. ${ }^{13} \mathrm{Com}-$ plications were classified into categories based on Systematized Nomenclature for Medicine-Clinical Terms codes (Supplementary Table 1) as follows: GI (bleeding, perforation) and non-GI (acute myocardial infarction [AMI], congestive heart failure $[\mathrm{CHF}]$, cerebrovascular accident [CVA; cerebral hemorrhage or infarction], and death). For death, 30-day in-hospital or emergency department death was analyzed by death domain of CDM.

\section{Statistical analysis}

Observational Health Data Sciences and Informatics analysis tools are embedded in the interactive analysis platform ATLAS. ATLAS version 2.7.2 was used herein, and we analyzed the platform of FEEDER-NET (Suwon, Korea), a health big-data platform based on Observational Health Data Sciences and Informatics-CDM supported by the Korean National Project. ${ }^{18}$ Data were presented as mean \pm standard deviation for normally distributed continuous variables and as number (percentage) for categorical variables. The chi-square or Fisher exact tests were used to

Table 2. Incidence Rate of Serious Complications within 30 Days of EGD by Age Group between 2012 and 2017

\begin{tabular}{|c|c|c|c|c|c|c|}
\hline Adverse events & $\begin{array}{c}2012 \\
A / B / C\end{array}$ & $\begin{array}{r}2013 \\
A / B / C\end{array}$ & $\begin{array}{c}2014 \\
A / B / C\end{array}$ & $\begin{array}{r}2015 \\
A / B / C\end{array}$ & $\begin{array}{c}2016 \\
A / B / C\end{array}$ & $\begin{array}{r}2017 \\
A / B / C\end{array}$ \\
\hline \multicolumn{7}{|l|}{ Bleeding } \\
\hline Subjects, No. & $\begin{array}{c}73,145 / \\
58,499 / 14,646\end{array}$ & $\begin{array}{c}68,029 / \\
53,613 / 14,416\end{array}$ & $\begin{array}{c}64,574 / \\
53,052 / 11,522\end{array}$ & $\begin{array}{c}60,302 / \\
46,487 / 13,815\end{array}$ & $\begin{array}{c}61,013 / \\
46,801 / 14,212\end{array}$ & $\begin{array}{c}60,225 / \\
39,580 / 20,645\end{array}$ \\
\hline Event cases, No. & $55 / 35 / 20$ & $60 / 29 / 31$ & $62 / 35 / 27$ & $40 / 19 / 21$ & $39 / 26 / 13$ & $51 / 18 / 33$ \\
\hline Proportion per 10,000 people & 7.5/6.5/13.7 & $8.8 / 5.4 / 21.5$ & $9.6 / 6.6 / 23.4$ & $6.6 / 4.1 / 15.2$ & $6.4 / 5.6 / 9.1$ & $8.5 / 4.5 / 16.0$ \\
\hline \multicolumn{7}{|l|}{ Perforation } \\
\hline Subjects, No. & $\begin{array}{c}73,197 / \\
58,529 / 14,668\end{array}$ & $\begin{array}{c}68,078 / \\
53,640 / 14,438\end{array}$ & $\begin{array}{c}64,609 / \\
53,071 / 11,538\end{array}$ & $\begin{array}{c}60,354 / \\
46,517 / 13,837\end{array}$ & $\begin{array}{c}61,075 / \\
46,847 / 14,228\end{array}$ & $\begin{array}{c}60,282 / \\
39,604 / 20,678\end{array}$ \\
\hline Event cases, No. & $7 / 3 / 4$ & $2 / 2 / 0$ & $2 / 1 / 1$ & $1 / 0 / 1$ & $1 / 0 / 1$ & $1 / 1 / 0$ \\
\hline Proportion per 10,000 people & $1.0 / 0.5 / 2.7$ & $0.3 / 0.4 / 0$ & $0.3 / 0.2 / 0.9$ & $0.2 / 0 / 0.7$ & $0.2 / 0 / 0.7$ & $0.2 / 0.3 / 0$ \\
\hline \multicolumn{7}{|l|}{ Cerebral hemorrhage or infarction } \\
\hline Subjects, No. & $\begin{array}{c}73,109 / \\
58,499 / 14,610\end{array}$ & $\begin{array}{c}68,012 / \\
53,622 / 14,390\end{array}$ & $\begin{array}{c}64,528 / \\
53,038 / 11,490\end{array}$ & $\begin{array}{c}60,302 / \\
46,503 / 13,799\end{array}$ & $\begin{array}{c}61,007 / \\
46,827 / 14,180\end{array}$ & $\begin{array}{c}60,229 / \\
39,594 / 20,635\end{array}$ \\
\hline Event cases, No. & $22 / 7 / 15$ & $12 / 4 / 8$ & $19 / 8 / 11$ & $20 / 7 / 13$ & $21 / 7 / 14$ & $13 / 4 / 9$ \\
\hline Proportion per 10,000 people & $3.0 / 1.2 / 10.3$ & $1.8 / 0.7 / 5.6$ & $2.9 / 1.5 / 9.6$ & $3.3 / 1.5 / 9.4$ & $3.4 / 1.5 / 9.9$ & $2.2 / 1.0 / 4.4$ \\
\hline \multicolumn{7}{|l|}{ Acute myocardial infarction } \\
\hline Subjects, No. & $\begin{array}{c}77,945 / \\
58,394 / 14,551\end{array}$ & $\begin{array}{c}67,805 / \\
53,499 / 14,306\end{array}$ & $\begin{array}{c}64,346 / \\
52,917 / 11,429\end{array}$ & $\begin{array}{c}60,072 / \\
46,364 / 13,708\end{array}$ & $\begin{array}{c}60,773 / \\
46,688 / 14,085\end{array}$ & $\begin{array}{c}60,066 / \\
39,535 / 20,531\end{array}$ \\
\hline Event cases, No. & $22 / 13 / 9$ & $30 / 14 / 16$ & $34 / 22 / 12$ & $32 / 15 / 17$ & $32 / 14 / 18$ & $19 / 4 / 15$ \\
\hline Proportion per 10,000 people & $3.0 / 2.2 / 6.2$ & $4.4 / 2.6 / 11.2$ & $5.3 / 4.2 / 10.5$ & $5.3 / 3.2 / 12.4$ & $5.3 / 3.0 / 12.8$ & $3.2 / 1.0 / 7.3$ \\
\hline \multicolumn{7}{|l|}{ Congestive heart failure } \\
\hline Subjects, No. & $\begin{array}{c}73,174 / \\
58,524 / 14,650\end{array}$ & $\begin{array}{c}68,072 / \\
53,646 / 14,426\end{array}$ & $\begin{array}{c}64,600 / \\
53,072 / 11,528\end{array}$ & $\begin{array}{c}60,337 / \\
46,510 / 13,827\end{array}$ & $\begin{array}{c}61,039 / \\
46,841 / 14,198\end{array}$ & $\begin{array}{c}60,251 / \\
39,602 / 20,649\end{array}$ \\
\hline Event cases, No. & $7 / 3 / 4$ & $7 / 1 / 6$ & $12 / 3 / 9$ & $5 / 1 / 4$ & $16 / 6 / 10$ & $11 / 1 / 10$ \\
\hline Proportion per 10,000 people & $1.0 / 0.5 / 2.7$ & $1.0 / 0.2 / 4.2$ & $1.9 / 0.6 / 7.8$ & $0.8 / 0.2 / 2.9$ & 2.6/1.3/7.0 & $1.8 / 0.3 / 4.8$ \\
\hline \multicolumn{7}{|l|}{ Death } \\
\hline Subjects, No. & $\begin{array}{c}73,203 / \\
58,533 / 14,670\end{array}$ & $\begin{array}{c}68,091 / \\
53,651 / 14,440\end{array}$ & $\begin{array}{c}64,617 / \\
53,079 / 11,538\end{array}$ & $\begin{array}{c}60,364 / \\
46,523 / 13,841\end{array}$ & $\begin{array}{c}61,085 / \\
46,856 / 14,229\end{array}$ & $\begin{array}{c}60,285 / \\
39,607 / 20,678\end{array}$ \\
\hline Event cases, No. & $25 / 12 / 13$ & $20 / 7 / 13$ & $14 / 8 / 6$ & $19 / 5 / 14$ & $10 / 2 / 8$ & $18 / 2 / 16$ \\
\hline Proportion per 10,000 people & $3.4 / 2.1 / 8.9$ & 2.9/1.3/9.0 & $2.2 / 1.5 / 5.2$ & $3.1 / 1.1 / 10.1$ & $1.6 / 0.4 / 5.6$ & $3.0 / 0.5 / 7.7$ \\
\hline
\end{tabular}

EGD, esophagogastroduodenoscopy; A, all age group; B, young age group (18-64 years); C, old age group ( $\geq 65$ years). 
examine relationships between categorical variables, and ttests were adopted to compare mean values of continuous variables. All p-values were two-tailed, and those less than 0.05 were considered statistically significant.

\section{RESULTS}

\section{Demographic characteristics of the study population}

A total of 387,647 patients who underwent EGD, 241,094 patients who underwent colonoscopy, and 89,059 patients who underwent colonoscopic polypectomy were captured from the study database between 2012 and 2017 (Table 1). The proportions of sex remained unchanged during the study period. The average age of patients significantly increased in the recent 3 years for EGD, colonoscopy, and colonoscopic polypectomy (all $\mathrm{p}<0.001$ ). The proportions of elderly patients also significantly increased in recent years for EGD, colonoscopy, and colonoscopic polypectomy (all $\mathrm{p}<0.001)$.

\section{Serious $\mathrm{GI}$ and non-GI complications in EGD}

Serious GI and non-GI complications within 30 days after diagnostic EGD were captured and classified by age group (Table 2). Pooled complication rates of bleeding and perforation were $7.9 / 10,000$ and $0.4 / 10,000$ persons, respectively. Bleeding rates were significantly lower in the younger group than in the older group; however, perforation rates were not significantly lower in the younger group (5.4 vs $16.5, \mathrm{p}<0.001$ and 0.2 vs $0.8, \mathrm{p}=0.230$, respectively). For non-GI complications, the pooled complication rates of CVA, AMI, and CHF were $2.8 / 10,000,4.4 / 10,000$, and 1.5/10,000 persons, respectively. The CVA, AMI, and CFS rates were significantly lower in the younger group than in the older group (1.2 vs $8.2,2.7$ vs 10.1 , and 0.5 vs 4.9 , all $\mathrm{p}<0.001$, respectively). The incidence of death within 30 days after EGD was 2.7/10,000 persons and was significantly lower in the younger group than in the older group $(1.2$ vs $7.8, \mathrm{p}<0.001)$. All these data are summarized in the schematic bar graph in Fig. 1A.

\section{Serious $\mathrm{Gl}$ and non-GI complications in colonoscopy}

Serious GI and non-GI complications within 30 days after diagnostic colonoscopy were captured and classified by age group (Table 3 ). Pooled complication rates of bleeding rate and perforation were 5.1/10,000 and 0.4/10,000 persons, respectively. Bleeding and perforation rates were not statistically different between the younger and older
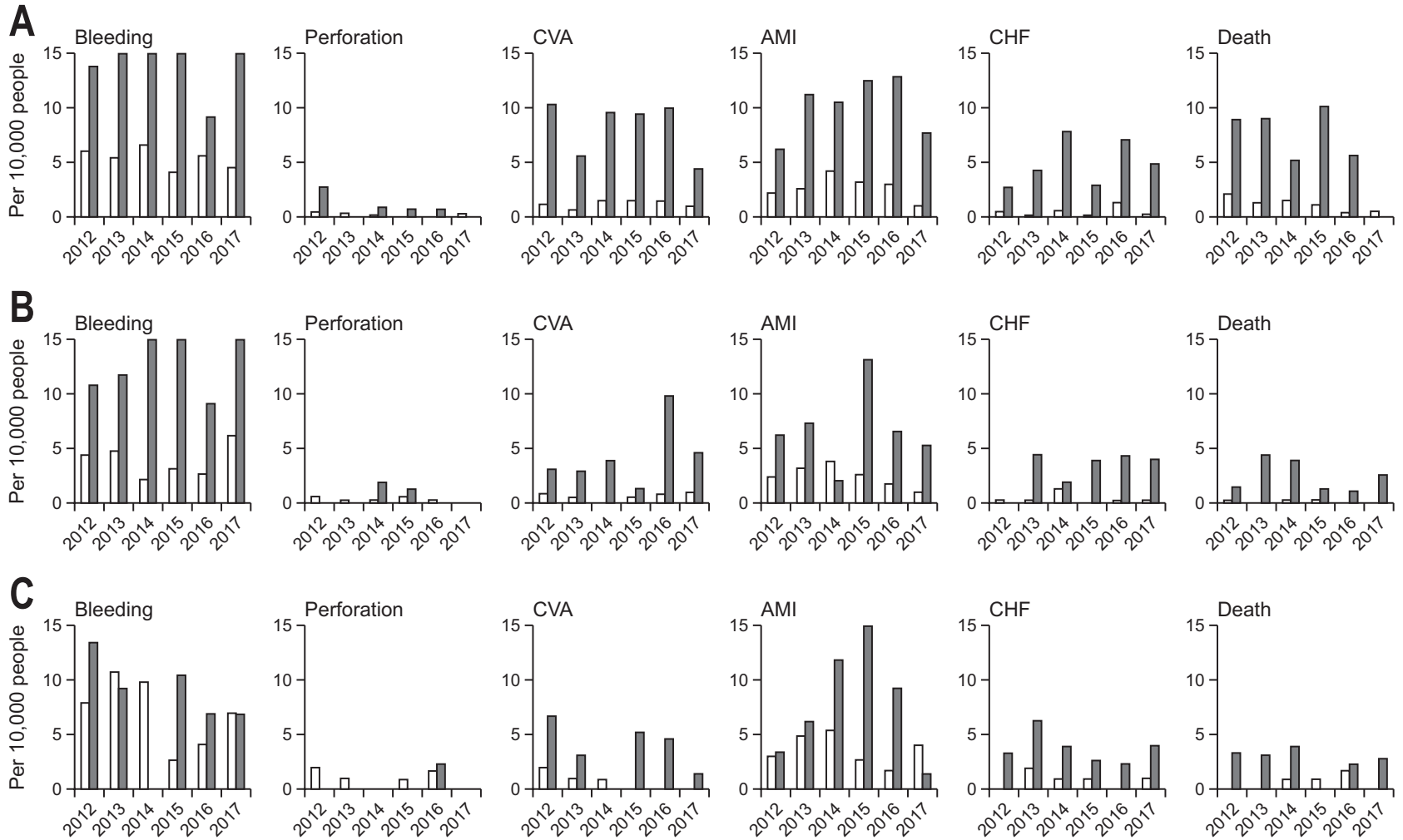

Fig. 1. Serious complication rates (event/10,000 persons) by age groups according to EGD (A), colonoscopy (B), and colonoscopic polypectomy (C). EGD, esophagogastroduodenoscopy; CVA, cerebrovascular accident; AMI, acute myocardial infarction; CHF, congestive heart failure. 
Table 3. Incidence Rate of Serious Complications within 30 Days of Diagnostic Colonoscopy between 2012 and 2017

\begin{tabular}{|c|c|c|c|c|c|c|}
\hline Adverse events & $\begin{array}{c}2012 \\
A / B / C\end{array}$ & $\begin{array}{c}2013 \\
A / B / C\end{array}$ & $\begin{array}{c}2014 \\
A / B / C\end{array}$ & $\begin{array}{c}2015 \\
A / B / C\end{array}$ & $\begin{array}{c}2016 \\
A / B / C\end{array}$ & $\begin{array}{c}2017 \\
\text { A/B/C }\end{array}$ \\
\hline \multicolumn{7}{|l|}{ Bleeding } \\
\hline Subjects, No. & $\begin{array}{c}40,460 / \\
33,990 / 6,470\end{array}$ & $\begin{array}{c}38,370 / \\
31,527 / 6,843\end{array}$ & $\begin{array}{c}36,804 / \\
31,654 / 5,150\end{array}$ & $\begin{array}{c}38,463 / \\
30,780 / 7,683\end{array}$ & $\begin{array}{c}42,786 / \\
33,559 / 9,227\end{array}$ & $\begin{array}{c}43,996 / \\
28,836 / 15,160\end{array}$ \\
\hline Event cases, No. & $22 / 15 / 7$ & $23 / 15 / 8$ & $16 / 7 / 9$ & $13 / 10 / 7$ & $19 / 9 / 10$ & $31 / 18 / 13$ \\
\hline Proportion per 10,000 people & $5.4 / 4.4 / 10.8$ & $6.0 / 4.8 / 11.7$ & 4.3/2.2/17.5 & $3.4 / 3.2 / 9.1$ & $4.4 / 2.7 / 10.8$ & $7.0 / 6.2 / 8.6$ \\
\hline \multicolumn{7}{|l|}{ Perforation } \\
\hline Subjects, No. & $\begin{array}{c}40,485 / \\
34,003 / 6,482\end{array}$ & $\begin{array}{c}38,388 / \\
31,537 / 6,851\end{array}$ & $\begin{array}{c}36,825 / \\
31,672 / 5,153\end{array}$ & $\begin{array}{c}38,479 / \\
30,790 / 7,689\end{array}$ & $\begin{array}{c}42,825 / \\
33,586 / 9,239\end{array}$ & $\begin{array}{c}44,049 / \\
28,861 / 15,188\end{array}$ \\
\hline Event cases, No. & $2 / 2 / 0$ & $1 / 1 / 0$ & $2 / 1 / 1$ & $3 / 2 / 1$ & $1 / 1 / 0$ & $0 / 0 / 0$ \\
\hline Proportion per 10,000 people & $0.5 / 0.6 / 0$ & $0.3 / 0.3 / 0$ & $0.5 / 0.3 / 1.9$ & $0.8 / 0.6 / 1.3$ & $0.2 / 0.3 / 0$ & $0 / 0 / 0$ \\
\hline \multicolumn{7}{|l|}{ Cerebral hemorrhage or infarction } \\
\hline Subjects, No. & $\begin{array}{c}40,457 / \\
33,993 / 6,464\end{array}$ & $\begin{array}{c}38,367 / \\
31,528 / 6,839\end{array}$ & $\begin{array}{c}36,808 / \\
31,662 / 5,146\end{array}$ & $\begin{array}{c}38,469 / \\
30,789 / 7,680\end{array}$ & $\begin{array}{c}42,801 / \\
33,574 / 9,227\end{array}$ & $\begin{array}{c}44,022 / \\
28,857 / 15,165\end{array}$ \\
\hline Event cases, No. & $5 / 3 / 2$ & $4 / 2 / 2$ & $2 / 0 / 2$ & $3 / 2 / 1$ & $12 / 3 / 9$ & $10 / 3 / 7$ \\
\hline Proportion per 10,000 people & $1.2 / 0.9 / 3.1$ & $1.0 / 0.6 / 2.9$ & $0.5 / 0 / 3.9$ & $0.8 / 0.6 / 1.3$ & $2.8 / 0.9 / 9.8$ & 2.3/1.0/4.6 \\
\hline \multicolumn{7}{|l|}{ Acute myocardial infarction } \\
\hline Subjects, No. & $\begin{array}{c}40,365 / \\
33,932 / 6,433\end{array}$ & $\begin{array}{c}38,291 / \\
31,474 / 6,817\end{array}$ & $\begin{array}{c}36,700 / \\
31,584 / 5,116\end{array}$ & $\begin{array}{c}38,326 / \\
30,701 / 7,625\end{array}$ & $\begin{array}{c}42,665 / \\
33,498 / 9,167\end{array}$ & $\begin{array}{c}43,901 / \\
28,814 / 15,087\end{array}$ \\
\hline Event cases, No. & $12 / 8 / 4$ & $15 / 10 / 5$ & $13 / 12 / 1$ & $18 / 8 / 10$ & $12 / 6 / 6$ & $11 / 3 / 8$ \\
\hline Proportion per 10,000 people & $3.0 / 2.4 / 6.2$ & $3.9 / 3.2 / 7.3$ & $3.5 / 3.8 / 2.0$ & 4.7/2.6/13.1 & $2.8 / 1.8 / 6.5$ & 2.5/1.0/5.3 \\
\hline \multicolumn{7}{|l|}{ Congestive heart failure } \\
\hline Subjects, No. & $\begin{array}{c}40,479 / \\
34,000 / 6,479\end{array}$ & $\begin{array}{c}38,395 / \\
31,543 / 6,852\end{array}$ & $\begin{array}{c}36,823 / \\
31,673 / 5,150\end{array}$ & $\begin{array}{c}38,474 / \\
30,791 / 7,683\end{array}$ & $\begin{array}{c}42,820 / \\
33,590 / 9,230\end{array}$ & $\begin{array}{c}44,033 / \\
28,857 / 15,176\end{array}$ \\
\hline Event cases, No. & $1 / 1 / 0$ & $4 / 1 / 3$ & $5 / 4 / 1$ & $3 / 0 / 3$ & $5 / 1 / 4$ & $7 / 1 / 6$ \\
\hline Proportion per 10,000 people & $0.2 / 0.3 / 0$ & $1.0 / 0.3 / 4.4$ & $1.4 / 1.3 / 1.9$ & $0.8 / 0 / 3.9$ & $1.2 / 0.3 / 4.3$ & $1.6 / 0.3 / 4.0$ \\
\hline \multicolumn{7}{|l|}{ Death } \\
\hline Subjects, No. & $\begin{array}{c}40,489 / \\
34,007 / 6,482\end{array}$ & $\begin{array}{c}38,398 / \\
31,546 / 6,852\end{array}$ & $\begin{array}{c}36,829 / \\
31,676 / 5,153\end{array}$ & $\begin{array}{c}38,488 / \\
30,798 / 7,690\end{array}$ & $\begin{array}{c}42,840 / \\
33,598 / 9,242\end{array}$ & $\begin{array}{c}44,050 / \\
28,862 / 15,188\end{array}$ \\
\hline Event cases, No. & $2 / 1 / 1$ & $3 / 0 / 3$ & $3 / 1 / 2$ & $2 / 1 / 1$ & $1 / 0 / 1$ & $4 / 0 / 4$ \\
\hline Proportion per 10,000 people & $0.5 / 0.3 / 1.5$ & $0.8 / 0 / 4.4$ & $0.8 / 0.3 / 3.9$ & $0.5 / 0.3 / 1.3$ & $0.2 / 0 / 1.1$ & $0.9 / 0 / 2.6$ \\
\hline
\end{tabular}

$A$, all age group; $B$, young age group (18-64 years); $C$, old age group ( $\geq 65$ years).

groups ( 3.9 vs $9.9, \mathrm{p}=0.059$ and 0.4 vs $0.4, \mathrm{p}>0.999$, respectively). For non-GI complications, the pooled complication rates of CVA, AMI, and CHF were 1.4/10,000, 3.4/10,000, and 1.0/10,000 persons, respectively. The CVA rate was significantly lower in the younger group than in the older group ( 0.7 vs $4.3, \mathrm{p}=0.038$ ), while the AMI and CHF rates were not statistically different between the younger and older groups ( 2.5 vs $6.7, \mathrm{p}=0.116$ and 0.4 vs $3.1, \mathrm{p}=0.079$, respectively). The incidence of death within 30 days after colonoscopy was $0.6 / 10,000$ persons and was lower in the younger group than in the older group without statistical significance ( 0.2 vs $2.4, \mathrm{p}=0.130)$. All these data are summarized in the schematic bar graph in Fig. 1B.

\section{Serious $\mathrm{Gl}$ and non-Gl complications in colonoscopic polypectomy}

Serious GI and non-GI complications within 30 days after colonoscopic polypectomy were captured and classified by age group (Table 4 ). Pooled complication rates of bleeding rate and perforation were 7.3/10,000 and $0.8 / 10,000$ persons, respectively. Bleeding and perforation rates were not statistically different between the younger and the older groups (7.0 vs 7.9, p>0.999 and 0.9 vs $0.4, \mathrm{p}>0.999$, respectively). For non-GI complications, pooled complication rates of CVA, AMI, CHF were 1.4/10,000, 4.6/10,000, and 1.7/10,000 persons, respectively. However, the CVA, AMI, and CHF rates were not statistically different between the young and the older groups ( 0.7 vs $3.3, p=0.648$; 3.6 vs $8.0, \mathrm{p}=0.651$; and 0.8 vs $4.0, \mathrm{p}=0.491$, respectively). The incidence of death within 30 days after colonoscopic polypectomy was $1.1 / 10,000$ persons and was lower in the younger group than in the older group without statistical significance ( 0.6 vs $2.5, \mathrm{p}=0.937$ ). All these data are summarized in the schematic bar graph in Fig. 1C.

\section{DISCUSSION}

In this large, population-based study, we evaluated the GI and non-GI complications of EGD, colonoscopy, and 
Table 4. Incidence Rate of Serious Complications within 30 Days of Colonoscopic Polypectomy between 2012 and 2017

\begin{tabular}{|c|c|c|c|c|c|c|}
\hline Adverse events & $\begin{array}{c}2012 \\
A / B / C\end{array}$ & $\begin{array}{c}2013 \\
A / B / C\end{array}$ & $\begin{array}{c}2014 \\
A / B / C\end{array}$ & $\begin{array}{c}2015 \\
A / B / C\end{array}$ & $\begin{array}{c}2016 \\
A / B / C\end{array}$ & $\begin{array}{c}2017 \\
A / B / C\end{array}$ \\
\hline \multicolumn{7}{|l|}{ Bleeding } \\
\hline Subjects, No. & $\begin{array}{c}13,122 / \\
10,126 / 2,996\end{array}$ & $\begin{array}{c}13,568 / \\
10,322 / 3,246\end{array}$ & $\begin{array}{c}13,746 / \\
11,179 / 2,567\end{array}$ & $\begin{array}{c}14,931 / \\
11,092 / 3,839\end{array}$ & $\begin{array}{c}16,427 / \\
12,057 / 4,370\end{array}$ & $\begin{array}{c}17,196 / \\
9,955 / 7,141\end{array}$ \\
\hline Event cases, No. & $12 / 8 / 4$ & $14 / 11 / 3$ & $11 / 11 / 0$ & $7 / 3 / 4$ & $8 / 5 / 3$ & $12 / 7 / 5$ \\
\hline Proportion per 10,000 people & $9.1 / 7.9 / 13.4$ & 10.3/10.7/9.2 & 8.0/9.8/0 & $4.7 / 2.7 / 10.4$ & 4.9/4.1/6.9 & 7.0/7.0/6.9 \\
\hline \multicolumn{7}{|l|}{ Perforation } \\
\hline Subjects, No. & $\begin{array}{c}13,132 / \\
10,135 / 2,997\end{array}$ & $\begin{array}{c}13,576 / \\
10,325 / 3,251\end{array}$ & $\begin{array}{c}13,755 / \\
11,184 / 2,571\end{array}$ & $\begin{array}{c}14,943 / \\
11,100 / 3,843\end{array}$ & $\begin{array}{c}16,443 / \\
12,064 / 4,379\end{array}$ & $\begin{array}{c}17,205 / \\
9,956 / 7,249\end{array}$ \\
\hline Event cases, No. & $2 / 2 / 0$ & $1 / 1 / 0$ & $0 / 0 / 0$ & $1 / 1 / 0$ & $3 / 2 / 1$ & $0 / 0 / 0$ \\
\hline Proportion per 10,000 people & $1.5 / 2.0 / 0$ & $0.7 / 1.0 / 0$ & $0 / 0 / 0$ & $0.7 / 0.9 / 0$ & $1.8 / 1.7 / 2.3$ & $0 / 0 / 0$ \\
\hline \multicolumn{7}{|l|}{ Cerebral hemorrhage or infarction } \\
\hline Subjects, No. & $\begin{array}{c}13,121 / \\
10,132 / 2,989\end{array}$ & $\begin{array}{c}13,565 / \\
10,320 / 3,245\end{array}$ & $\begin{array}{c}13,752 / \\
11,182 / 2,570\end{array}$ & $\begin{array}{c}14,929 / \\
11,094 / 3,835\end{array}$ & $\begin{array}{c}16,429 / \\
12,058 / 4,371\end{array}$ & $\begin{array}{c}17,196 / \\
9,955 / 7,241\end{array}$ \\
\hline Event cases, No. & $4 / 2 / 2$ & $2 / 1 / 1$ & $1 / 1 / 0$ & $2 / 0 / 2$ & $2 / 0 / 2$ & $1 / 0 / 1$ \\
\hline Proportion per 10,000 people & $3.0 / 2.0 / 6.7$ & $1.5 / 1.0 / 3.1$ & $0.7 / 0.9 / 0$ & $1.3 / 0 / 5.2$ & $1.2 / 0 / 4.6$ & $0.6 / 0 / 1.4$ \\
\hline \multicolumn{7}{|l|}{ Acute myocardial infarction } \\
\hline Subjects, No. & $\begin{array}{c}13,083 / \\
10,112 / 2,971\end{array}$ & $\begin{array}{c}13,531 / \\
10,294 / 3,237\end{array}$ & $\begin{array}{c}13,704 / \\
11,156 / 2,548\end{array}$ & $\begin{array}{c}14,875 / \\
11,070 / 3,805\end{array}$ & $\begin{array}{c}16,373 / \\
12,022 / 4,351\end{array}$ & $\begin{array}{c}17,130 / \\
9,927 / 7,203\end{array}$ \\
\hline Event cases, No. & $4 / 3 / 1$ & $7 / 5 / 2$ & $9 / 6 / 3$ & $9 / 3 / 6$ & $6 / 2 / 4$ & $5 / 4 / 1$ \\
\hline Proportion per 10,000 people & $3.1 / 3.0 / 3.4$ & $5.2 / 4.9 / 6.2$ & $6.6 / 5.4 / 11.8$ & $6.1 / 2.7 / 15.8$ & $3.7 / 1.7 / 9.2$ & $2.9 / 4.0 / 1.4$ \\
\hline \multicolumn{7}{|l|}{ Congestive heart failure } \\
\hline Subjects, No. & $\begin{array}{c}13,131 / \\
10,136 / 2,995\end{array}$ & $\begin{array}{c}13,575 / \\
10,324 / 3,251\end{array}$ & $\begin{array}{c}13,755 / \\
11,184 / 2,571\end{array}$ & $\begin{array}{c}14,939 / \\
11,097 / 3,842\end{array}$ & $\begin{array}{c}16,434 / \\
12,060 / 4,374\end{array}$ & $\begin{array}{c}17,200 / \\
9,955 / 7,245\end{array}$ \\
\hline Event cases, No. & $1 / 0 / 1$ & $4 / 2 / 2$ & $2 / 1 / 1$ & $2 / 1 / 1$ & $1 / 0 / 1$ & $5 / 1 / 4$ \\
\hline Proportion per 10,000 people & $0.8 / 0 / 3.3$ & $2.9 / 1.9 / 6.2$ & $1.5 / 0.9 / 3.9$ & $1.3 / 0.9 / 2.6$ & $0.6 / 0 / 2.3$ & $2.9 / 1.0 / 5.5$ \\
\hline \multicolumn{7}{|l|}{ Death } \\
\hline Subjects, No. & $\begin{array}{c}13,133 / \\
10,236 / 2,997\end{array}$ & $\begin{array}{c}13,578 / \\
10,327 / 3,251\end{array}$ & $\begin{array}{c}13,756 / \\
11,185 / 2,571\end{array}$ & $\begin{array}{c}14,944 / \\
11,101 / 3,843\end{array}$ & $\begin{array}{c}16,443 / \\
12,064 / 4,379\end{array}$ & $\begin{array}{c}17,205 / \\
9,956 / 7,249\end{array}$ \\
\hline Event cases, No. & $1 / 0 / 1$ & $1 / 0 / 1$ & $2 / 1 / 1$ & $1 / 1 / 0$ & $3 / 2 / 1$ & $2 / 0 / 2$ \\
\hline Proportion per 10,000 people & $0.8 / 0 / 3.3$ & $0.7 / 0 / 3.1$ & $1.5 / 0.9 / 3.9$ & $0.7 / 0.9 / 0$ & $1.8 / 1.7 / 2.3$ & $1.2 / 0 / 2.8$ \\
\hline
\end{tabular}

$A$, all age group; $B$, young age group (18-64 years); $C$, old age group ( $\geq 65$ years).

colonoscopic polypectomy using a well-defined HIRA-NPS database over a 6-year period. There had been few population-based studies conducted on the exact rates of GI and non-GI complications of these procedures, and previous studies on colonoscopy have been mostly reported from Western countries. ${ }^{4,7,12,19-22}$ The most important finding of the present study was that the rates of serious non-GI and GI complications are higher in the elderly population than in the younger population. The overall complication rates of each endoscopy procedure were similar to those of recent population-based studies despite their heterogeneous time interval (15 days vs 30 days) and targeted population (e.g., screening, diagnostic, therapeutic purpose, or mixed) for endoscopy. ${ }^{4,7,12,21-23}$ Serious GI and non-GI complication rates remain very low in the overall study population; however, the elderly population experienced about three times more complications than those of the younger population when undergoing diagnostic EGD or colonoscopy. As global aging increases and a growing need for GI endoscopy in elderly patients becomes increasingly inevitable in the real world, the safety of GI endoscopy in elderly patients is an ongoing concern for physicians. ${ }^{24-27}$ Therefore, our study is unique in that the safety of EGD, colonoscopy, and colonoscopic polypectomy is reported with respect to both GI and non-GI complications using a populationbased database from Asia.

EGD is generally known to be safe and well-tolerated in the elderly; ${ }^{22,25-28}$ however, its risk is largely related to comorbidity and sedation during the procedure. To our knowledge, there has been no population-based study performed to evaluate GI and non-GI complications after EGD in the elderly population. In our study, the complication rates of GI bleeding, CVA, AMI, CHF, and death were significantly higher in the elderly group. However, the perforation rate remained very low in both age groups, which may be explained by our assessment of only diagnostic EGD. Well-known risk factors of EGD, such as comorbidity, sedative medications, and susceptibility levels for sedatives, have been reported in previous non-populationbased research ${ }^{22,25}$ and could have a greater influence on 
elderly than younger patients. In the real world, diagnostic EGD is performed in elderly patients with comorbidity and ongoing medication use as necessary, which may increase the complication rate of EGD in the real-world data relative to that in well-controlled prospective clinical trials. Therefore, physicians should pay more attention to the indications of EGD in elderly patients.

Complications of colonoscopy have been more extensively studied than those of EGD. However, there are limited data available for comparing elderly and nonelderly populations. In a systematic review and meta-analysis, ${ }^{23}$ elderly patients had a higher risk of complications during and after colonoscopy, like our study. However, direct comparison with our study is difficult due to the heterogeneity of studies included in the meta-analysis, different population and different definition of complications. The recent population-based study reported complication rates of colonoscopy in all age groups, ${ }^{7}$ which showed similar ranges of complication rates with our study. In previous studies, the observed complication rates were generally two to three times higher in the elderly group than young age group, which is consistent with our findings. ${ }^{7,23,25,29} \mathrm{~A}$ population-based study reported a slightly higher perforation rate in the elderly population from the United States (92 vs 69 per 100,000 persons) $;{ }^{30}$ however, few populationbased studies have been performed in Asian countries. The overall perforation rate of colonoscopy has been reported to be lower in Asian countries than in Western countries, ${ }^{31,32}$ which is also consistent with our results. Overall, it is difficult to compare the perforation rate between young and old populations in Asian and Western countries because of the low incidence of perforation; therefore, further comparative population-based investigation is necessary. Elderly patients are more vulnerable to colonoscopyrelated complications irrespective of the region and clinical setting.

Colonoscopic polypectomy has been commonly performed and effectively applied for removing adenoma to prevent colorectal cancer, ${ }^{33-36}$ yet its GI complication rate is at least 4-fold higher than that of colonoscopy alone. ${ }^{21,22,30}$ As the complications of colonoscopic polypectomy have mainly focused on GI events, there have been no population-based studies to capture its non-GI complication rates. ${ }^{11,22}$ In our study, GI complication rates of colonoscopic polypectomy were not significantly different between young and old patients. However, non-GI complication rates of colonoscopic polypectomy were about 3-fold higher in elderly patients than in young patients. These results indicate that non-GI complications are more problematic than GI complications in elderly patients undergoing colonoscopic polypectomy. Future studies should focus not only on GI complications, but also non-GI complications in elderly patients undergoing colonoscopic polypectomy.

Our study has several limitations. As we used HIRANPS, an administrative claims-based database, it is difficult to assess detailed information, such as comorbid diseases or endoscopist and facility characteristics, or detailed clinical data such as indications of EGD or colonoscopy. Furthermore, we could not assess the data of EGD or colonoscopy performed to the patients not under the medical insurance program, which means the possibility of previous endoscopy experience not captured by HIRA-NPS data. But, these cases may be minimal, if any, considering most endoscopies are reimbursed in South Korea. Also, our study included only outpatients, which lead to possible data loss of patients with severe comorbidities. In addition, as inpatient colonoscopy and colonoscopic polypectomy were excluded from this study, patients with more severe comorbidities may be excluded. Our data should be cautiously interpreted as it is not reasonable to directly compare complication rates between colonoscopy group and colonoscopic polypectomy groups because they are different cohorts. The 30-day post-procedure observation period may be arbitrary but is borrowed from the surgical literature and other population-based research from the United States. ${ }^{7}$ Data collection was retrospective, and there are inevitable data quality issues with conversion of HIRA data to the CDM database. However, excellent data quality has been validated in recent studies using CDM databases. ${ }^{37,38}$

In conclusion, despite GI endoscopy carrying a low risk of complication, elderly patients experienced a roughly 3 -fold higher rate of GI and non-GI complications. Physicians should pay attention to non-GI complication risks as well as GI complication risks of GI endoscopy when treating elderly patients.

\section{CONFLICTS OF INTEREST}

No potential conflict of interest relevant to this article was reported.

\section{ACKNOWLEDGEMENTS}

This work was supported by a grant from Kyung Hee University in 2018 (KHU-20181044). 


\section{AUTHOR CONTRIBUTIONS}

Study concept and design: H.I.K., J.M.C. Data acquisition: M.S.K., J.Y.Y. Data analysis and interpretation: H.I.K., J.M.C. Drafting of the manuscript; critical revision of the manuscript for important intellectual content, study supervision: H.I.K., J.M.C. Statistical analysis: H.I.K. Obtained funding: J.M.C. Administrative, technical, or material support: M.S.K., J.Y.Y. Approval of final manuscript: all authors.

\section{ORCID}

Ha Il Kim

Jin Young Yoon

Min Seob Kwak

Jae Myung Cha https://orcid.org/0000-0002-5471-7790

https://orcid.org/0000-0002-5280-0443

https://orcid.org/0000-0002-8988-7423

https://orcid.org/0000-0001-9403-230X

\section{REFERENCES}

1. Rabeneck L, Saskin R, Paszat LF. Onset and clinical course of bleeding and perforation after outpatient colonoscopy: a population-based study. Gastrointest Endosc 2011;73:520523.

2. Whitlock EP, Lin J, Liles E, et al. Screening for colorectal cancer: an updated systematic review [Internet]. Rockville: Agency for Healthcare Research and Quality (US); c2008 [cited 2020 Jun 15]. Available from: https://www.ncbi.nlm. nih.gov/books/NBK35179/.

3. Sharma VK, Nguyen CC, Crowell MD, Lieberman DA, de Garmo P, Fleischer DE. A national study of cardiopulmonary unplanned events after GI endoscopy. Gastrointest Endosc 2007;66:27-34.

4. Cotton PB, Eisen GM, Aabakken L, et al. A lexicon for endoscopic adverse events: report of an ASGE workshop. Gastrointest Endosc 2010;71:446-454.

5. Kothari ST, Huang RJ, Shaukat A, et al. ASGE review of adverse events in colonoscopy. Gastrointest Endosc 2019;90:863-876.

6. Kim SY, Moon CM, Kim MH, et al. Impacts of age and sedation on cardiocerebrovascular adverse events after diagnostic GI endoscopy: a nationwide population-based study. Gastrointest Endosc 2020;92:591-602.

7. Wang L, Mannalithara A, Singh G, Ladabaum U. Low rates of gastrointestinal and non-gastrointestinal complications for screening or surveillance colonoscopies in a populationbased study. Gastroenterology 2018;154:540-555.

8. Quine MA, Bell GD, McCloy RF, Charlton JE, Devlin HB, Hopkins A. Prospective audit of upper gastrointestinal en- doscopy in two regions of England: safety, staffing, and sedation methods. Gut 1995;36:462-467.

9. Wolfsen HC, Hemminger LL, Achem SR, et al. Complications of endoscopy of the upper gastrointestinal tract: a single-center experience. Mayo Clin Proc 2004;79:1264-1267.

10. Zubarik R, Eisen G, Mastropietro C, et al. Prospective analysis of complications 30 days after outpatient upper endoscopy. Am J Gastroenterol 1999;94:1539-1545.

11. Levin TR, Zhao W, Conell C, et al. Complications of colonoscopy in an integrated health care delivery system. Ann Intern Med 2006;145:880-886.

12. Reumkens A, Rondagh EJ, Bakker CM, Winkens B, Masclee AA, Sanduleanu S. Post-colonoscopy complications: a systematic review, time trends, and meta-analysis of population-based studies. Am J Gastroenterol 2016;111:1092-1101.

13. Kim JA, Yoon S, Kim LY, Kim DS. Towards actualizing the value potential of Korea Health Insurance Review and Assessment (HIRA) data as a resource for health research: strengths, limitations, applications, and strategies for optimal use of HIRA Data. J Korean Med Sci 2017;32:718-728.

14. Overhage JM, Ryan PB, Reich CG, Hartzema AG, Stang PE. Validation of a common data model for active safety surveillance research. J Am Med Inform Assoc 2012;19:54-60.

15. Hripcsak G, Duke JD, Shah NH, et al. Observational Health Data Sciences and Informatics (OHDSI): opportunities for observational researchers. Stud Health Technol Inform 2015;216:574-578.

16. Qureshi WA, Zuckerman MJ, Adler DG, et al. ASGE guideline: modifications in endoscopic practice for the elderly. Gastrointest Endosc 2006;63:566-569.

17. Kedia P, Waye JD. Colon polypectomy: a review of routine and advanced techniques. J Clin Gastroenterol 2013;47:657665.

18. Park RW. The Distributed Research Network, Observational Health Data Sciences and Informatics, and the South Korean Research Network. Korean J Med 2019;94:309-314.

19. Sieg A, Hachmoeller-Eisenbach U, Eisenbach T. Prospective evaluation of complications in outpatient GI endoscopy: a survey among German gastroenterologists. Gastrointest Endosc 2001;53:620-627.

20. Rabeneck L, Paszat LF, Hilsden RJ, et al. Bleeding and perforation after outpatient colonoscopy and their risk factors in usual clinical practice. Gastroenterology 2008;135:18991906.

21. Arora G, Mannalithara A, Singh G, Gerson LB, Triadafilopoulos $\mathrm{G}$. Risk of perforation from a colonoscopy in adults: a large population-based study. Gastrointest Endosc 2009;69(3 Pt 2):654-664.

22. Warren JL, Klabunde CN, Mariotto AB, et al. Adverse events after outpatient colonoscopy in the Medicare population. Ann Intern Med 2009;150:849-857. 
23. Day LW, Kwon A, Inadomi JM, Walter LC, Somsouk M. Adverse events in older patients undergoing colonoscopy: a systematic review and meta-analysis. Gastrointest Endosc 2011;74:885-896.

24. Travis AC, Pievsky D, Saltzman JR. Endoscopy in the elderly. Am J Gastroenterol 2012;107:1495-1501.

25. ASGE Standards of Practice Committee, Chandrasekhara V, Early DS, et al. Modifications in endoscopic practice for the elderly. Gastrointest Endosc 2013;78:1-7.

26. Razavi F, Gross S, Katz S. Endoscopy in the elderly: risks, benefits, and yield of common endoscopic procedures. Clin Geriatr Med 2014;30:133-147.

27. Levy I, Gralnek IM. Complications of diagnostic colonoscopy, upper endoscopy, and enteroscopy. Best Pract Res Clin Gastroenterol 2016;30:705-718.

28. ASGE Standards of Practice Committee, Ben-Menachem T, Decker GA, et al. Adverse events of upper GI endoscopy. Gastrointest Endosc 2012;76:707-718.

29. Kim SY, Kim HS, Park HJ. Adverse events related to colonoscopy: global trends and future challenges. World J Gastroenterol 2019;25:190-204.

30. Gatto NM, Frucht H, Sundararajan V, Jacobson JS, Grann VR, Neugut AI. Risk of perforation after colonoscopy and sigmoidoscopy: a population-based study. J Natl Cancer Inst 2003;95:230-236.

31. Teoh AY, Poon CM, Lee JF, et al. Outcomes and predictors of mortality and stoma formation in surgical management of colonoscopic perforations: a multicenter review. Arch Surg 2009;144:9-13.

32. Shi X, Shan Y, Yu E, et al. Lower rate of colonoscopic perforation: 110,785 patients of colonoscopy performed by colorectal surgeons in a large teaching hospital in China. Surg Endosc 2014;28:2309-2316.

33. Lee BI, Hong SP, Kim SE, et al. Korean guidelines for colorectal cancer screening and polyp detection. Clin Endosc 2012;45:25-43.

34. Lee SH, Shin SJ, Park DI, et al. Korean guideline for colonoscopic polypectomy. Clin Endosc 2012;45:11-24.

35. Rex DK, Boland CR, Dominitz JA, et al. Colorectal cancer screening: recommendations for physicians and patients from the U.S. Multi-Society Task Force on Colorectal Cancer. Am J Gastroenterol 2017;112:1016-1030.

36. Wolf AM, Fontham ET, Church TR, et al. Colorectal cancer screening for average-risk adults: 2018 guideline update from the American Cancer Society. CA Cancer J Clin 2018;68:250-281.

37. Hripcsak G, Suchard MA, Shea S, et al. Comparison of cardiovascular and safety outcomes of chlorthalidone vs hydrochlorothiazide to treat hypertension. JAMA Intern Med 2020;180:542-551.

38. Wang Q, Reps JM, Kostka KF, et al. Development and validation of a prognostic model predicting symptomatic hemorrhagic transformation in acute ischemic stroke at scale in the OHDSI network. PLoS One 2020;15:e0226718. 